\title{
Efecto de la suplementación con hidratos de carbono y proteínas sobre el rendimiento físico deportivo en futbolistas amateur y profesionales: revisión sistemática
}

\section{Protein and carbohydrate supplementation on physical performance in amateur and professional football players: systematic review}

\author{
Vanessa G. Temimilpa-Sánchez ${ }^{a}$, Luis Delgado-Olivares ${ }^{b}$, José A. Ariza-Ortega $^{c}$ y Araceli \\ Ortiz-Polo $^{d}$
}

\begin{abstract}
:
Soccer is a very popular team game worldwide, considered as an intermittent sport due to the intense movements of short duration with high energy demand, causing fatigue and decreased performance during exercise. This review aims to analyze current research on the effects of the intake of supplements with HCO, PT and protein derivatives on performance in soccer players and identify those that had a significant impact. A systematic online review was carried out based on articles published in PubMed, Scielo, Redalyc, Elsevier and Dialnet using keywords such as "football", "supplementation", "carbohydrates", "protein", "physical performance", "ergogenic" and "recovery". At the beginning, 33 studies were found that performed interventions in soccer players with supplements of HCO and PT, of which only 11 showed to have a significant impact on physical tests and biochemical markers. The interventions that had a better impact on the performance and recovery of the soccer players, used SN composed of HCO and PT. Sports supplementation must be individualized and it's important to be accompanied by a nutritionist to advise on the consumption of SN that maximize the benefits.
\end{abstract}

\section{Keywords:}

Soccer, supplementation, carbohydrates, protein, physical performance, fatigue, recovery.

\section{Resumen:}

El fútbol es un juego de equipo muy popular mundialmente, considerado como un deporte intermitente debido a los movimientos intensos de corta duración con alta demanda energética, causante de fatiga y disminución del rendimiento durante el juego y entrenamientos. Analizar investigaciones actuales sobre los efectos de la ingesta de suplementos con HCO, proteína y derivados proteicos sobre el rendimiento en futbolistas e identificar aquellos que tuvieron un mayor impacto. Se realizó una revisión sistemática en la red con base a artículos publicados en PubMed, Scielo, Redalyc, Elsevier y Dialnet utilizando palabras claves como "football", "supplementation", "carbohydrates", "protein", "physical performance", "ergogenic" y "recovery". Se encontraron en un inicio 33 estudios que realizaron intervenciones en futbolistas con suplementos de HCO y PT, de los cuales solo 11 mostraron tener algún impacto significativo sobre las pruebas físicas y marcadores bioquímicos. Las intervenciones que tuvieron un mayor impacto en el rendimiento y recuperación de los futbolistas utilizaron SN compuestos de HCO y PT en conjunto. La suplementación deportiva debe ser individualizada y es importante el acompañamiento de un profesional en nutrición que asesore sobre el consumo de SN que maximicen los beneficios.

\section{Palabras Clave:}

Fútbol, suplementación, hidratos de carbono, proteína, rendimiento, fatiga, recuperación.

\footnotetext{
a Universidad Autónoma del Estado de Hidalgo, Instituto de Ciencias de la Salud, https://orcid.org/0000-0002-5531-1434,

Email: te277007@uaeh.edu.mx

b Universidad Autónoma del Estado de Hidalgo, Instituto de Ciencias de la Salud, http://orcid.org/0000-0002-3506-8393

c Universidad Autónoma del Estado de Hidalgo, Instituto de Ciencias de la Salud, http://orcid.org/0000-0002-2163-4593

d Autor de Correspondencia, Universidad Autónoma del Estado de Hidalgo, Instituto de Ciencias de la Salud, https://orcid.org/0000-00015561-2221, E-mail: araceli_ortiz4208@uaeh.edu.mx
}

Fecha de recepción: 11/10/2020, Fecha de aceptación: 09/12/2020, Fecha de publicación: 05/06/2021 


\section{Introducción}

El fútbol es considerado un deporte de equipo, y una de las prácticas deportivas más populares mundialmente, cuenta con aproximadamente 200 millones de jugadores registrados en 186 países. ${ }^{1}$ Es considerado como un deporte intermitente, en donde los jugadores realizan movimientos de baja intensidad durante la mayor parte del juego, y realizan hasta 250 movimientos intensos de corta duración, y pueden recorrer hasta $13 \mathrm{~km}$ durante un partido, todo ello implica que los jugadores tengan una alta demanda de energía, siendo una causante importante de fatiga producida durante el juego. ${ }^{2}$

A medida que aumenta el nivel competitivo, la intensidad del esfuerzo y el número de partidos que se juegan por temporada también se incrementan. En cada partido se llevan a cabo alrededor de 30 a 50 carreras de 10 a 15 metros de intensidad muy elevada, provocando una importante disminución de las reservas energéticas del jugador, las cuales deben ser repuestas mediante la ingesta correcta de alimentos. ${ }^{3}$

El consumo adecuado de nutrientes en los jugadores asegura el mantenimiento de un estado de nutrición correcto, un óptimo rendimiento y una pronta recuperación de lesiones o prevención de las mismas. ${ }^{4}$

Para poder proporcionar estrategias nutricionales a futbolistas, es necesario conocer las demandas energéticas que cada uno tiene, y cuáles son los sustratos que se utilizan durante los partidos. ${ }^{5}$ Es bien sabido que el glucógeno hepático y muscular son las reservas de hidratos de carbono ( $\mathrm{HCO}$ ) más importantes para la producción de energía, por lo que una adecuada ingesta de éste sustrato previo, durante y posterior a los entrenamientos garantizará la energía necesaria para un rendimiento óptimo y pronta recuperación muscular, ya que según varios estudios realizados, si no se restaura por completo el contenido de glucógeno muscular, se limita la habilidad de los jugadores para hacer un sprint y saltar, actividades esenciales durante un partido. ${ }^{5}$

Otro sustrato de gran importancia son las proteínas (PT), que tienen un papel crucial durante las adaptaciones producidas en respuesta al ejercicio, y debido a que durante el juego se realizan acciones intensas como correr, acelerar, desacelerar y saltar es probable que existan lesiones en las fibras musculares, disminuyendo el rendimiento. ${ }^{6,7}$

Algunas intervenciones de recuperación nutricional sugieren que una ingesta de HCO y PT mayor a la habitual puede participar en procesos de regeneración muscular y de esta forma acelerar la recuperación en este contexto surgen los suplementos nutricionales (SN), definidos como "productos que se toman vía oral y que contienen un ingrediente dietético como vitaminas, minerales, hierbas u otros ingredientes botánicos, aminoácidos y sustancias como enzimas y metabolitos" según la Sociedad Internacional de Nutrición del Deporte $(2018)^{8}$, con el fin de lograr un beneficio específico para la salud y/o el rendimiento.

Una buena alimentación y el uso de SN pueden ser de mucha utilidad en la mejora del rendimiento físico deportivo en este deporte. ${ }^{9}$ Estudios previos han demostrado que una suplementación con PT apresura su renovación en el músculo esquelético al interferir con su degradación en condiciones donde existe un balance negativo de proteínas como sucede durante los partidos de fútbol. ${ }^{6}$ En base a los estudios revisados, no existe gran evidencia científica que muestre cuáles son los suplementos PT o con $\mathrm{HCO}$ que realmente tienen un impacto positivo en el rendimiento de jugadores de fútbol a cualquier nivel; por lo que la siguiente revisión tiene como objetivo analizar las investigaciones actuales sobre los efectos de la ingesta de SN con HCO, PT y derivados proteicos sobre el rendimiento físico en estos deportistas e identificar aquellos que tuvieron un mayor impacto durante los entrenamientos y partidos.

\section{Métodos}

El método utilizado ha sido la búsqueda exhaustiva a través de bases de datos como PubMed, Scielo, Redalyc, Elservier, Dialnet, Academia.edu; además de revistas y libros especializadas en deporte, nutrición y medicina deportiva.

La revisión bibliográfica se realizó en lengua hispana e inglesa, y las palabras clave fueron football o soccer and supplementation, carbohidrates, protein, physical performance, athletic performance, resistance exercise, dietary supplement, ergogenic, recovery o muscle mass.

Se excluyeron aquellos documentos publicados antes del año 2015, aquellos que no consideraran HCO o PT como SN, y los que utilizaron como muestra otro tipo de deportistas que no fueran futbolistas.

\section{Resultados}

Los estudios revisados utilizaron como muestra futbolistas masculinos amateur y profesionales pertenecientes a los clubes de primera y segunda división, a excepción de un artículo en donde la muestra fue con mujeres universitarias que practicaban el deporte. ${ }^{10}$

En la revisión, se encontró que la mayoría de los artículos midieron el efecto directo que tienen los SN sobre el rendimiento físico. ${ }^{10-14}$ 
Tabla 1. Características de los estudios incluidos.

\begin{tabular}{|c|c|c|c|c|}
\hline Autores (año) & Muestra & Suplementación & Pruebas y test & Resultados \\
\hline Naclerio et al (2015). ${ }^{13}$ & $\begin{array}{l}\text { Edad: } 24 \pm 3.7 \\
\mathrm{n}=16 \text { hombres jugadores de fútbol } \\
\text { amateur }\end{array}$ & $\begin{array}{l}\text { Ingesta de } 500 \mathrm{~mL} \text { de suplemento } \\
\text { con solo HCO }(53 \mathrm{~g}) \text {, combinado } \\
(53 \mathrm{~g} \text { HCO, } 14.5 \mathrm{~g} \text { PT, 5g GLUT, } \\
1.5 \mathrm{~g} \text { L-carnitina) o placebo (PL). }\end{array}$ & $\begin{array}{l}\text { Peso, talla, } \mathrm{VO} 2_{\max }, 90 \mathrm{~min} \text { sprints } \\
\text { intermitentes, RPE, IRS, MAS, } \\
\text { muestras de sangre (marcadores de } \\
\text { fatiga incluyendo IL- } 6, \mathrm{Mb} \text { y CK). }\end{array}$ & $\begin{array}{l}\text { Ninguna mejoró rendimiento, pero } \\
\text { comparando la combinada con las } \\
\text { otras atenuó la fatiga al no haber un } \\
\text { aumento de la IL-6 tras una hora; } \\
\text { en la de HCO solo, aumentaron } \\
\text { neutrófilos y monocitos después de } \\
90 \text { min de sprints. }\end{array}$ \\
\hline Rodríguez et al (2015). ${ }^{10}$ & $\begin{array}{l}\text { Edad: } 23 \pm 5 \\
n=18 \text { futbolistas universitarias }\end{array}$ & $\begin{array}{l}\text { Suplementación } \text { de } \beta-\text { alanina } \\
(2.4 \mathrm{~g} / \text { día) por } 30 \text { días } \\
\text { consumiendo } 3 \text { "grajeas" de } 800 \\
\text { mg durante el día. }\end{array}$ & $\begin{array}{l}\text { Pre y post test, antropometría, } 3 \\
\text { pruebas de Wingate } 30 \text { seg, LD } 3 \\
\text { veces, entrenamiento (sprints, } \\
\text { velocidad, distancia). }\end{array}$ & $\begin{array}{l}\text { Incremento en el rendimiento y } \\
\text { potencia en } 3 \text { pruebas de Wingate } \\
\text { sucesivas; no hubo diferencia en } \\
\text { LD. }\end{array}$ \\
\hline Albert et al. (2015). ${ }^{14}$ & $\begin{array}{l}\mathrm{n}=24 \text { jugadores de } 1^{\text {ra }} \text { división } \\
\text { Control } \mathrm{n}=16\end{array}$ & $\begin{array}{l}\text { Suplementación por } 6 \text { días con } \\
\text { HMB }(3 \mathrm{~g}), \mathrm{HMB}(3 \mathrm{~g})+\text { Creatina } \\
\text { (3g) y PL }(6 \mathrm{~g} \mathrm{HCO}) \text { disueltos en } \\
250 \mathrm{~mL} \text { de agua con el desayuno. }\end{array}$ & $\begin{array}{l}\text { Antropometría, pruebas de } \\
\text { velocidad, muestra de sangre en } \\
\text { ayunas de LD y CK, potencia y } \\
\text { fatiga. }\end{array}$ & $\begin{array}{l}\text { HMB y HMB }+ \text { creatina } \\
\text { incrementan el rendimiento } \\
\text { anaeróbico y reducen niveles de } \\
\text { CK y LD, además de una } \\
\text { disminución en el porcentaje de } \\
\text { grasa. }\end{array}$ \\
\hline Guzel et al. (2015). ${ }^{17}$ & $\begin{array}{l}\text { Edad: } 17-19 \\
\mathrm{n}=26 \text { hombres jugadores } \\
\text { profesionales. }\end{array}$ & $\begin{array}{l}\text { Ingesta de un vaso de jugo de fruta } \\
\text { con } 3 \text { o } 4 \mathrm{~g} \text { de L-Carnitina. }\end{array}$ & $\begin{array}{l}\text { FC, prueba de velocidad de } 8- \\
10 \mathrm{~km} / \mathrm{h} \text {, muestra de sangre (GSH, } \\
\text { TBARs). }\end{array}$ & $\begin{array}{l}\text { Dosis de } 3 \text { y } 4 \mathrm{~g} \text { de L- Carnitina } \\
\text { mostró un incremento en GSH y } \\
\text { disminución de TBARs, redujo el } \\
\text { estrés oxidativo y tuvo efecto } \\
\text { antioxidante; se incrementó la } \\
\text { velocidad máxima al correr y se } \\
\text { disminuyó la FC }\end{array}$ \\
\hline Filiz et al. (2016). ${ }^{19}$ & $\begin{array}{l}\text { Edad: } 15 \pm 2.2 \\
\mathrm{n}=21 \text { jugadores 1ra división }\end{array}$ & $\begin{array}{l}\text { Ingesta de bebida isotónica con } 32 \mathrm{~g} \\
\text { de } \mathrm{HCO} \text { en } 500 \mathrm{~mL} \text { de agua }(\mathrm{n}=11) \\
\text { o } 500 \mathrm{~mL} \text { de agua en botellas } \\
\text { oscuras }(\mathrm{n}=10) \text {. }\end{array}$ & $\begin{array}{l}\text { Extracción de sangre en ayunas } \\
\text { antes, } 2 \text { y } 24 \mathrm{~h} \text { después de la } \\
\text { prueba, medición de troponina, } \\
\text { mioglobina, CK, LD; carrera de } 60- \\
80 \text { min. }\end{array}$ & $\begin{array}{l}\text { Efecto protector y menor daño } \\
\text { muscular que el placebo durante la } \\
\text { prueba y el periodo de } \\
\text { recuperación. }\end{array}$ \\
\hline Ferreira et al (2017). ${ }^{20}$ & $\begin{array}{l}\text { Edad: } 24 \pm 4 \\
n=10 \text { hombres jugadores de fútbol } \\
\text { amateur }\end{array}$ & $\begin{array}{l}\text { Suplementación de } 200 \mathrm{~mL} \text { cada } 10 \\
\text { min de ejercicio con HCO } \\
\text { (maltodextrina) o HCO + proteína } \\
4: 1+\text { antioxidantes (CPA) diluido } \\
\text { en agua a } 6-8 \% \text {. }\end{array}$ & $\begin{array}{l}3 \text { sesiones de ejercicio aeróbico en } \\
\text { cicloergómetro de } 50 \text { min, FC, } \\
\text { muestra sanguínea (CK, TBARS, } \\
\text { Glucosa). }\end{array}$ & $\begin{array}{l}\text { Ambas previnieron la baja de } \\
\text { niveles de Glucosa durante } \\
\text { ejercicio. Sólo CPA previno estrés } \\
\begin{array}{l}\text { oxidativo, y una pronta } \\
\text { recuperación de glucógeno } \\
\text { muscular. }\end{array}\end{array}$ \\
\hline Poulios et al (2018). ${ }^{16}$ & $\begin{array}{l}\text { Edad: } 20.6 \pm 1.1 \\
\mathrm{n}=20 \text { hombres de } 1^{\text {ra }}, 2^{\text {da }} \text { y } 3^{\text {ra }} \\
\text { división. }\end{array}$ & $\begin{array}{l}\text { Suplemento con } 80 \mathrm{~g} \text { de PT de leche } \\
\text { concentrada o placebo } \\
\text { (maltodextrina) en días de juego, y } \\
\text { en entrenamientos } 20 \mathrm{~g} \text { de caseína o } \\
\text { placebo. }\end{array}$ & $\begin{array}{l}\text { Antropometría, } \mathrm{VO}_{2} \text { max máximo } \\
\text { latido, prueba Yo-Yo, pruebas de } \\
\text { velocidad y rendimiento (sprints y } \\
\text { saltos), muestra de sangre (CK, } \\
\text { TBARs, PCR, GSH, Hb, HTO). }\end{array}$ & $\begin{array}{l}\text { Suplemento de proteína facilita la } \\
\text { recuperación en los entrenamientos } \\
\text { al disminuir la fatiga } \\
\text { neuromuscular y respuestas } \\
\text { inflamatorias y acelerar la } \\
\text { regeneración del músculo. }\end{array}$ \\
\hline
\end{tabular}




\begin{tabular}{|c|c|c|c|c|}
\hline Chtourou et al (2019). ${ }^{21}$ & $\begin{array}{l}\text { Edad: } 21.2 \pm 1.2 \\
\mathrm{n}=19 \text { estudiantes jugadores y } \\
\text { físicamente activos }\end{array}$ & $\begin{array}{l}\text { Suplementación con } 250 \mathrm{~mL} \text { de } \\
\text { bebida energética (cafeína, taurina, } \\
\left.\text { HCO, proteína y } \mathrm{B}_{6}\right) \text { o PL (sin } \\
\text { cafeína, taurina y con acesulfame } \\
\text { K) antes y después de las pruebas } \\
(500 \mathrm{~mL}) \text { y una comida } \\
\text { estandarizada. }\end{array}$ & $\begin{array}{l}\text { Toma de glucosa, RPE, test de } \\
\text { estado de alerta y velocidad de } \\
\text { reacción motora, test de Wingate } \\
\text { con cicloergómetro por } 30 \mathrm{seg} \text {. }\end{array}$ & $\begin{array}{l}\text { El estado de alerta y velocidad de } \\
\text { reacción mejoraron tras la ingesta } \\
\text { de la bebida energética (Red Bull), } \\
\text { se mejoró el rendimiento en la } \\
\text { prueba de Wingate y cambios } \\
\text { favorables de RPE reflejados en un } \\
\text { mejor rendimiento, además de un } \\
\text { aumento en la glucemia. }\end{array}$ \\
\hline Smith et al (2019). ${ }^{22}$ & $\begin{array}{l}\text { Edad: } 21 \pm 1.8 \\
n=15 \text { jugadores universitarios } \\
\text { Control } n=8\end{array}$ & $\begin{array}{l}\text { Suplementación con cápsulas de } \beta \text { - } \\
\text { alanina o PL (maltodextrina) } 6.4 \\
\text { g/día durante } 6 \text { semanas. }\end{array}$ & $\begin{array}{l}\text { Antropometría y composición } \\
\text { corporal, prueba de sprints (6 sets } \\
\text { de } 30 \text { seg y } 30 \text { seg de descanso } \\
\text { entre cada uno), 1RM de sentadilla } \\
\text { y prensa de pecho, IR, USE y LSE; } \\
\text { determinación de LD y FC. }\end{array}$ & $\begin{array}{l}\text { Suplemento con } \beta \text { - alanina mostró } \\
\text { un mínimo impacto en la } \\
\text { composición corporal y en la fuerza } \\
\text { muscular, hubo una mejora poco } \\
\text { significativa en pruebas de sprints y } \\
\text { ejercicios de alta intensidad en } \\
\text { intervalos. }\end{array}$ \\
\hline Sun, L. (2020). ${ }^{15}$ & $\begin{array}{l}\text { Edad: no mencionada } \\
\mathrm{n}=16 \text { hombres jugadores de fútbol } \\
\text { amateur }\end{array}$ & $\begin{array}{l}\text { Ingesta de } 800 \mathrm{~mL} \text { de bebida con } \\
\text { Oligosacáridos + suero de leche } \\
(25 \mathrm{~g}) 4: 1 \text { y placebo }\end{array}$ & $\begin{array}{l}\text { Prueba de } \mathrm{VO}_{2 \max } \text {, prueba de } \\
\text { capacidad anaeróbica, prueba de } \\
\text { rendimiento físico. }\end{array}$ & $\begin{array}{l}\text { El momento en el que se hace la } \\
\text { reposición de oligosacáridos más el } \\
\text { suero de PT, la capacidad aeróbica } \\
\text { y anaeróbica en el fútbol mejora y } \\
\text { se promueve la recuperación. }\end{array}$ \\
\hline Toro et al. (2020). ${ }^{18}$ & $\begin{array}{l}\text { Edad: } 22.36 \pm 1.36 \\
n=32 \text { jugadores de fútbol amateur } \\
\text { Control } n=16\end{array}$ & $\begin{array}{l}\text { Ingesta de } 200 \mathrm{mg} / \text { día de TC o PL } \\
200 \mathrm{mg} \text { de lactosa en polvo durante } \\
30 \text { días. }\end{array}$ & $\begin{array}{l}\text { Extracción sanguínea (ácido úrico, } \\
\text { TG, HDL, LDL, Glucosa, CT, } \\
\text { GGT y Hb), antropometría, } \\
\text { evaluación nutricional, prueba de } \\
\text { ergoespirometría (velocidad, } \mathrm{VO}_{2} \\
\text { absoluto y relativo, FC). }\end{array}$ & $\begin{array}{l}\text { La suplementación diaria modificó } \\
\text { parámetros en pruebas } \\
\text { hematológicas (aumento en } \mathrm{Hb} \text {, } \\
\text { glucosa, ácido úrico y creatina y } \\
\text { disminución de } \mathrm{FC} \text { ) y en la } \\
\text { ergoespirometría (aumento de } \\
\mathrm{VO}_{2 \mathrm{max} \text { y oxígeno) pudiendo }} \\
\text { utilizarse como ayuda ergogénica } \\
\text { para un mejor rendimiento y } \\
\text { recuperación pronta sin efectos } \\
\text { negativos. } \\
\text { No hubo cambios significativos en } \\
\text { los valores antropométricos. }\end{array}$ \\
\hline
\end{tabular}


En cuanto a los tipos de SN encontrados, se utilizaron aquellos que incluyeran $\mathrm{HCO}$ de bajo o alto índice glicémico (IG); PT, aminoácidos (AA) y sustancias nitrogenadas tales como caseína, PT de suero, PT de hidrolizada, monohidrato de creatina, $\beta$-alanina, $\beta$-hidroxi$\beta$ - metilbutirato (HMB), L-Carnitina, Glutamina, entre otros. ${ }^{10-17}$

El rango de edad en las muestras utilizadas en los estudios es de 15 a 28 años. El 54.5\% de los estudios incorporaban "mejorar el rendimiento", como principal razón al consumir los $\mathrm{SN}$, el resto buscaba disminuir el daño muscular (18.8\%), atenuar la fatiga., prevenir el daño oxidativo u otras razones $(26.7 \%)$.

Las pruebas y test utilizados para medir el efecto del SN incluían sprints repetidos intermitentes (IRS), velocidad aeróbica máxima (MAS), 1 repetición máxima (1RM), prueba de carrera intermitente (IR), tasa de esfuerzo percibido (RPE) ejercicios de resistencia y velocidad, pruebas de fuerza en tren superior (USE) e inferior (LSE); indicadores bioquímicos de creatinkinasa (CK), Interleucina-6 (IN-6), lactato deshidrogenasa (LD), glucosa, glutatión (GSH), ácido tiobarbitúrico (TBARs), hemoglobina $(\mathrm{Hb})$, hematocrito $(\mathrm{HTO})$, PT C reactiva $(\mathrm{PCR})$, colesterol $(\mathrm{CT})$, triglicéridos $(\mathrm{TG})$, ácido úrico, lipoproteína de alta densidad (HDL) y de baja densidad (LDL), gamma-glutamil transferasa (GGT); antropometría; frecuencia cardiaca (FC) y el volumen máximo de oxígeno $\left(\mathrm{VO}_{2}\right.$ max $)$. Las intervenciones que mostraron tener un efecto significativo en el rendimiento de los futbolistas fueron aquellas que suplementaron con compuestos de $\mathrm{HCO}$ y $\mathrm{AA}$ y $\mathrm{SN}$ únicamente proteicos como de $\beta$ - alanina, HMB y HMB con creatina. ${ }^{10,11,14}$ Otro SN poco utilizado que tuvo efectos positivos en los marcadores bioquímicos y en el rendimiento fue una microalga denominada Tetrasekmis chuii (TC) compuesta de PT, HCO, AA, minerales y ácidos grasos. ${ }^{18} \mathrm{La}$ tabla 1 recoge los autores, el año, el tipo de SN utilizado y la administración del mismo, así como la forma en la que se midieron las variables (tests y pruebas) y los resultados de los trabajos más relevantes de la revisión.

\section{Discusión}

El fútbol es un deporte de alta intensidad y duración, ya que un partido dura entre 90 y 120 min, y diversos estudios demuestran que el rendimiento físico disminuye después de éste tiempo; ${ }^{23-25}$ por lo que para poder optimizar el rendimiento durante partidos y entrenamientos, es necesario que los jugadores consuman la suficiente cantidad de HCO y PT que se requieren para el trabajo y las adaptaciones fisiológicas, de lo contrario, una deficiencia en los mismos se reflejaría en pérdida de masa muscular, de densidad ósea y aumentaría el riesgo de lesiones, y como consecuencia un menor rendimiento deportivo. ${ }^{26}$

Las estrategias nutricionales para éstos atletas se centran principalmente en suministrar suficiente energía para optimizar las reservas antes del ejercicio y para proveer energía durante el mismo, ${ }^{27}$ sin embargo, conocer el rendimiento es más complejo en juegos de equipo, debido a las distintas posiciones o roles de cada jugador, por lo que el enfoque nutricional es más difícil de definir. Un jugador gasta hasta $1,600 \mathrm{kcal}$ durante un partido por lo que los requerimientos energéticos se deberán calcular individualmente de acuerdo a la posición del jugador,

Las recomendaciones para futbolistas de élite en $\mathrm{HCO}$ de $7-10 \mathrm{~g} / \mathrm{kg}$ para recuperación de un entrenamiento de alta intensidad, una ingesta de $1.4-1.7 \mathrm{~g} / \mathrm{kg}$ de proteína y un $20-30 \%$ de la ingesta calórica total de grasas $^{8}$.

Tras la revisión de varios artículos, se encontró que la forma más común de suplementar con $\mathrm{HCO}$ en futbolistas es en bebidas isotónicas, las cuales se recomiendan antes y durante el partido para mantener la glucemia estable durante el ejercicio intermitente y prevenir deshidratación, ${ }^{28}$ coincidiendo con los estudios de Harper y Rusell en 2016, quienes hallaron que al ingerir cada 15 minutos durante un partido una bebida con $\mathrm{HCO}$, se mantiene la glucemia durante el ejercicio. ${ }^{24,29}$

En el estudio de Filiz y colaboradores en 2016, hallaron que la suplementación con una bebida isotónica con $32 \mathrm{~g}$ de HCO tiene un efecto protector contra el daño muscular durante el ejercicio prolongado al haber disminuido marcadores de lesión muscular como CK y LD. Fue un estudio aleatorizado doble ciego lo que aumenta la validez del mismo; por otra parte, la suplementación no fue individualizada, y los jugadores tienen distintas posiciones y requerimientos, lo que limita los resultados de este estudio, y habría que especificar las dosis de cada jugador. ${ }^{19}$

Stevenson y colaboradores en 2017 realizaron una intervención en donde la muestra se dividió en tres grupos, uno con SN de alto IG (maltodextrina), otro con bajo IG (isomaltosa) y un placebo; los resultados mostraron que ninguno atenuó la fatiga, pero la isomaltosa disminuyó la epinefrina y causó menos malestar abdominal. ${ }^{12}$

Al comparar el estudio anterior con el de Poilios en 2018 se rescata que utilizaron la maltodextrina como PL, y caseína como grupo control, obteniendo mejores resultados, ya que al adicionar $\mathrm{PT}$ se redujo la fatiga neuromuscular y las respuestas inflamatorias, permitiendo acelerar la regeneración muscular utilizando como indicadores el conteo de glóbulos blancos, granulocitos, CK, TBARS, carbonilos proteicos, GSH y la actividad total antioxidante (TAC). ${ }^{16}$ 
En una intervención con 7 jugadores se investigaron los efectos sobre el rendimiento en el ejercicio anaeróbico tras la ingesta de electrolitos con AA y diferentes concentraciones de HCO. Se trató de un estudio aleatorizado doble ciego, en donde la ingesta de AA no tuvo efecto en las pruebas de rendimiento, sin embargo, al adicionar $\mathrm{HCO}$ y electrolitos, se observaron mejoras al realizar sprints y mayor altura en los saltos. ${ }^{11}$ En esta intervención la validez de la muestra está limitada debido a la poca cantidad de sujetos, y a que no se individualizaron las cantidades; sin embargo, los resultados coinciden con otra investigación, en donde se comprobó un mejor rendimiento en sprints repetitivos tras consumir el suplemento con $\mathrm{HCO}$ y electrolitos. ${ }^{12}$

Estos datos no concuerdan con el estudio de Naclerio en 2015, donde analizaron los efectos de la ingesta antes, durante y después de realizar $90 \mathrm{~min}$ de sprints intermitentes de un compuesto de $\mathrm{HCO}$ y AA o sólo $\mathrm{HCO}$, sobre el rendimiento, fatiga e indicadores metabólicos de daño muscular ración en comparación con el PL, ya que en esta intervención ninguno de los grupos mostró mejoría en el rendimiento, pero el tiempo de fatiga se retrasó en el SN combinado. ${ }^{13}$

En cuanto al diseño, el estudio es similar al de Albert y colaboradores en 2015, con una muestra aleatorizada de grupos y con los SN administrados de forma doble ciego. ${ }^{14}$ Respecto a la validez de la muestra, el estudio de Nacleiro en 2015 tiene una muestra con jugadores amateur, mientras que el otro es una muestra con jugadores profesionales de primera división, por lo que los requerimientos de los jugadores son muy variables en ambos casos.

En éste último estudio, se investigaron los efectos del consumo de creatina y de $\mathrm{HMB}$, el cual tiene un papel ergogénico al reducir la respuesta inflamatoria por el ejercicio, influir en la fuerza y la masa magra al actuar como agente anticatabólico, minimizando la proteólisis y daño celular. Los datos sugieren que la suplementación puede aumentar el rendimiento y reducir niveles de CK y LD. ${ }^{13,14}$ Como factor limitante, se tendría que analizar si los resultados se pueden extrapolar a jugadores de nivel no profesional.

Ferreira y colaboradores en 2016 investigaron el efecto de un SN combinado de HCO, PT y antioxidantes (vitamina $\mathrm{C}$ y $\mathrm{E}$ de 45 y $10 \mathrm{mg}$ respectivamente). En este estudio encontraron mejoras significativas en la reducción de estrés oxidativo y recuperación de depósitos de glucógeno de una forma más rápida. Sin embargo, la muestra solo fue de 10 sujetos, por lo que puede ser una limitación importante en el artículo. ${ }^{20}$ Los resultados obtenidos en el estudio son similares a los que obtuvieron Guzel y colaboradores en el 2015, donde 26 jugadores profesionales redujeron niveles de estrés oxidativo tras consumir SN de L-Carnitina, demostrando un efecto antioxidante. ${ }^{17}$

De igual forma, Chtourou y colaboradores en 2019 realizaron una intervención aleatorizada doble ciego con 19 jugadores, encontrando efectos positivos al suplementar con $250 \mathrm{~mL}$ de una bebida energética compuesta por HCO, PT, cafeína, y biotina antes y después de las pruebas físicas, obteniendo un mejor rendimiento durante la prueba de esfuerzo, mayor velocidad de reacción y mayor estado de alerta. ${ }^{21}$

Rodríguez en el año 2015 incluyó en su estudio futbolistas universitarias, y al solo ser una muestra con mujeres limita la comparación con otros artículos, sin embargo, los resultados obtenidos a través de la suplementación con $2.4 \mathrm{~g}$ diarios de $\beta$ - alanina fueron positivos, obteniendo un incremento en el rendimiento y la potencia en pruebas de sprints, saltos, velocidad y distancia. $^{10}$

Se observaron efectos favorables en la capacidad aeróbica y anaeróbica en jugadores amateur de fútbol y una recuperación más óptima tras la intervención de Sun $L$ en 2020, en donde se suplementaron $800 \mathrm{~mL}$ de una bebida compuesta con oligosacáridos y suero de leche en proporción 4:1, midiendo los resultados con pruebas de $\mathrm{VO}_{2}$ max y pruebas de rendimiento físico. ${ }^{15}$

Más allá de los requerimientos nutricionales para mantener la salud, los nutrientes adicionales o SN como PT pueden coadyuvar a la mejor ejecución de los roles de cada jugador durante el juego. ${ }^{30}$

Estos últimos artículos, incluyeron $\mathrm{PT}$ y compuestos nitrogenados como suplementación, tal es el caso de la creatina, que ha tenido un efecto sobre el rendimiento y la mejora en sprints repetidos según varias investigaciones, como la realizada por Santesteban en el 2015, basándose en que éste aumenta la rapidez de resíntesis de fosfocreatina y ATP entre cada sprint; ${ }^{30}$ también se ha demostrado un efecto en prevenir la disminución de potencia. ${ }^{31}$ La $\beta$ - alanina fue otro de los SN proteicos más usados en los estudios considerados, debido a que se ha probado su eficacia en diversas investigaciones, donde en ejercicios de alta intensidad como el fútbol permite mejorar la potencia media, ${ }^{10,32}$ y se obtienen mejores resultados al acompañarlo con $\mathrm{HCO}$ y proteínas según el Instituto Australiano del Deporte (AIS). ${ }^{33}$

El Comité Olímpico Internacional (COI), agrupó aquellos SN que tienen suficiente evidencia sobre los beneficios que proporcionen, entre ellos se encuentran la cafeína por su efecto estimulante recomendando de 3-6 $\mathrm{mg} / \mathrm{kg} 60 \mathrm{~min}$ previos a la AF; la creatina de inicio $20 \mathrm{~g} /$ día dividido en 4 dosis iguales por 5-7 días y de mantenimiento 3-5 g/ día en una dosis y combinarla con fuentes de PT y HCO; $\beta$ - alanina de $65 \mathrm{mg} / \mathrm{kg}$ diariamente 
durante 10-12 semanas; SN de HCO ingerir 30-60 g/hr para atenuar hormonas de estrés; glutamina $2 \times 5 \mathrm{~g}$ después de competiciones y HMB dosis de $3 \mathrm{~g} /$ día para mejorar fuerza y masa muscular ${ }^{34}$.

\section{Conclusión}

Suplementar HCO en deportes de resistencia de alta intensidad permite mantener la disponibilidad de glucógeno y glucosa, retrasando la fatiga y manteniendo el rendimiento. Es recomendable el consumo de $\mathrm{HCO}$ de alto IG como maltodextrina durante el ejercicio por su rápida disponibilidad; y de bajo IG como galactosa e isomaltosa previo al mismo. Los HCO administrados en el fútbol tienen un efecto en pruebas de sprints, saltos y velocidad, reflejados en un mejor rendimiento.

Las PT permiten la recuperación y mantenimiento de los tejidos, y en el fútbol se utilizan SN como caseína, PT aislada de suero de leche, AA esenciales (EAA) por su rápida absorción, L-Taurina, glutamina, $\mathrm{HMB}$, creatina y $\beta$ alanina.

Los SN con PT e HCO en conjunto disminuyen de forma más efectiva marcadores de daño muscular y dolor después del ejercicio, mejoran la fuerza, la resistencia y la recuperación de glucógeno muscular.

Los biomarcadores que permiten monitorear el rendimiento y la fatiga en el fútbol son el LD, interleucina 6 (IL-6), PCR, cortisol, testosterona, LDH, mioglobina, urea y ácido úrico.

La suplementación deportiva debe de ser individualizada y específica para el rol de cada jugador, por lo que es importante el acompañamiento de un profesional en nutrición que asesore al equipo sobre el consumo de $\mathrm{SN}$ que maximicen los beneficios y minimicen los riesgos.

\section{Conflicto de intereses}

de intereses.

Los autores declaramos que no tenemos conflicto

\section{Referencias}

[1] Castellano J. Relación entre indicadores de rendimiento y el éxito en el fútbol profesional. Revista Iberoamericana de Psicología del Ejercicio y el Deporte. 2018; 13 (1), 41-49. [Fecha de consulta 27 de mayo de 2020]. ISSN: 1886-8576. Disponible en: https://www.redalyc.org/articulo.oa?id=3111/311153534004

[2] Bush M, Barnes D, Archer B, Hogg y P. S. Bradley. Evolution of match performance parameters for various playing positions in the English Premier League. Hum Mov Sci. 2015; 39: 1-11.

[3] Collins J, McCall A, Bilsborough J y Maughan R. Nutrición futbolística: ¿es hora de un nuevo consenso? 2017; 1577-1578.

[4] Abbey EL, Wright CJ y Kirkpatrick CM. Prácticas de nutrición y conocimiento entre jugadores de fútbol de la NCAA División III. Revista de la Sociedad Internacional de Nutrición Deportiva. 2017; 14 (1): 13 .
[5] Almendáriz A, Sánchez J. Ayudas ergogénicas en jugadores de fútbol profesional: Una revisión sistemática. Revista de educación, motricidad e investigación. 2018; 11, 83-97.

[6] Mohr M, Draganidis D, Chatzinikolaou A, Barbero-Álvarez J, Castagna C, Douroudos I, Avloniti A, Margeli A, Papassotiriou I, Flouris, A.D et al. Muscle damage, inflammatory, immune and performance responses to three football games in one week in competitive male players. Eur. J. Appl. Physiol. 2016; 116, 179-193.

[7] Draganidis D, Chatzinikolaou A, Avloniti A, Barbero-Álvarez J, Mohr M, Malliou P, Gourgoulis V, Deli C, Douroudos I, Margonis K. Recovery kinetics of knee flexor and extensor strength after a football game. PLoS ONE. 2015; 10.

[8] Sociedad internacional de Nutrición del Deporte (ISSN). Revisión de nutrición deportiva y de ejercicio del ISSN: investigación y recomendaciones. Revista de la sociedad internacional de nutrición deportiva. 2018; 7:7

[9] Mata F, Sánchez-Oliver J, Domínguez R \& Villegas J. Suplementación en el deporte: directrices desde la responsabilidad profesional. Habilidad motriz: Revista de ciencias de la actividad física y del deporte. 2018; (51), 4-12.

[10] Rodríguez F, Ormeño A, Rivera P, Tapia V y Montero C. Efectos de la suplementación con $\beta$ - alanina en tests de Wingate en jugadoras universitarias de fútbol femenino. Nutr Hops. 2015; 31(1):430-435.

[11] Krings B, Rountree J, McAllister M, Cummings P, Peterson T, Fountain B. y Smith J. Effects of acuate carbohydrate ingestion on anaerobic exercise performance. Journal of the International Society of Sports Nutrition. 2016; 13:40. DOI 0.1186/s12970-016-0152-9

[12] Stevenson E, Watson A, Theis S, Holz A, Harper L y Russell M. A comparison of isomaltulose versus maltodextrin ingestion during soccer- specific exercise. 2017

[13] Naclerio F, Larumbe E, Cooper R, Allgrove J y Earnest C. A MultiIngridient containing HCO, Proteins L-Glutamine and L-Carnitine attenuates fatigue perception with no effect on performance, muscle damage or immunity in soccer players. Journal Plus One. 2015; (4): 117. DOI: :10.1371/journal.pone.0125188

[14] Albert F, Morente S, Ortega F, Castillo M. y Gutiérrez A. Eficacia de la suplementación con $\beta$-hydroxy- $\beta$-methylbutyrate (HMB) en el deporte: actualización e implicación práctica. Nutr Hosp. 2015; 32(1):20-33

[15] Sun J. Effect of Whey Protein on Aerobic Exercise Ability Football Players. Matrix Sci Med. 2020; 3:19-21. DOI: 10.4103/MTSM.MTSM_23_19.

[16] Poulios, et al. Post-game high protein intake may improve recovery of football-specific performance during a congested game fixture: results from the pro-football study. Nutrients. 2018;(10), 494, DOI: 10.3390/nu10040494.

[17] Guzel N, Orer G, Sezen F y Cevher S. Effects of acute L-carnitine supplementation on nitric oxide production and oxidative stress after exhaustive exercise in young soccer players. J Sports med phys fitness. $2015 ; 55: 9-15$

[18] Toro V, Siquier-Coll V, Bartolomé I, Robles-Gil y M Maynar-Mariño M. Effects of tetraselmis chuii microalgae supplementation on ergospirometric, haematological and biochemical parameters in amateur soccer players. Int. J.Enviroment.Res.Public health. 2020;17,6885; doi:10.3390/ijerph17186885.

[19] Filiz F, Cayci B, Yaman M, Karagan S, Gonulates S, Ipekoglu G, Fatmantur E. The effects of the intake of an isotonic sports drink before orienteering competitions on skeletal muscle damage. J. Phys. Ther. 2016; 28 : 3200-3204.

[20] Ferreira D, Tavares L, Leal L, Santos G, Lima A, Pereira M, Santos A. Carbohydrates plus protein reduces oxidative stress after single bout of aerobic exercise. Journal of Human Sport and Exercise. 2016; 2(4): 1208-1219. DOI: 10.14198/jhse.2017.124.07. 
[21] Chtourou H, Trabelsi K, Ammar A, Shephard R y Bragazzi N. Effects of an "Energy Drink" on short-term maximal performance, reaction times, psychological and physiological parameters: insights from a randomized double-blind, placebo-controlled, counterbalanced crossover trial. Nutrints. 2019; 11, 992; doi:103390/nu11050992

[22] Smith C, Harty P, Stecker R y Kerksick C. A pilot study to examine the impact of beta-alanine supplementation on anaerobic exercise performance in collegiate athletes. Sports. 2019;7,231; doi:10.3390/sports7110231.

[23] Harper LD, Stevenson EJ, Rollo I, Russell M. The influence of a 12\% carbohydrate-electrolyte beverage on self-paced soccer specific exercise performance. J Sci Med Sport. 2017.

[24] Harper LD, Briggs MA, McNamee G, West DJ, Kilduff LP, Stevenson E y Russell M. Physiological and performance effects of carbohydrate gels consumed prior to the extra-time period of prolonged simulated soccer match-play. J Sci Med Sport. 2016a; 19:509-514

[25] Harper LD, Hunter R, Parker P et al. Test-retest reliability of physiological and performance responses to $120 \mathrm{~min}$ of simulated soccer match-play. J Strength Cond Res. 2016b; 30:3178-3186

[26] Jenner S, Buckley G, Belski R, Devlin B y Forsyth A. Dietary intakes of professional and semi-professional team sport Athletes don't meet sport nutrition recommendations. Nutrients. 2019; 11, 1160. DOI $10.3390 /$ nu 11051160 .

[27]American College of Sports Medicine. Nutrition and Athletic Performance. Medicine and Science in Sports and Exercise. 2016; 543568. DOI: $10.1249 / \mathrm{MSS} .0000000000000852$

[28] Thomas DT, Erdman KA, Burke LM. American College of Sports Medicine Joint Position Statement. Nutrition and athletic performance. Med Sci Sports Exerc. 2016; 48:543-568

[29] Russell M, Sparkes W, Northeast J, Kilduff LP. Responses to a 120 min reserve team soccer match: a case study focusing on the demands of extra-time. J Sport Sci. 2015a; 33:2133-2139

[30] Williams C, Rollo I. Carbohydrate Nutrition and team sport performance. Sport Med. 2015; 45: 13-22. DOI: 10.1007/s40279-0150399-3

[31] Santesteban M, Ibáñez J. Ayudas ergogénicas en el deporte. Nutr Hosp. 2017; 34: 204-215. Doi: http://dx.doi.org/10.20960/nh.997

[32] Claudino G, Mezêncio B, Amaral S et al. Creatine monohydrate supplementation on lower-limb muscle power in Brazilian elite soccer players. J Int Soc Sports Nutr 2015; 11:32.

[33] Australian Institute of Sport. Programa de suplementación para deportistas. Sports Magazine - Revista de entrenamiento. 2017; 10 (3) [Fecha de consulta 3 de Agosto de 2020]. Disponible en: http://www.isde.com.ar/ojs/index.php/isdesportsmagazine/article/view $\underline{\text { File/52/71 }}$

[34] Maughan R, et al. IOC consensus statement: dietary supplement and the high-performance athlete. Sports Med. 2018; 52: 439-455. DOI 0.1136/bjsports-2018-099027. 